OPEN ACCESS

Edited by:

Rafael De Prado,

Universidad de Córdoba, Spain

Reviewed by:

Isabel Calha,

Instituto Nacional de Investigação

Agrária e Veterinária, Portugal

Pedro Jacob Christoffoleti,

University of São Paulo, Brazil

*Correspondence:

Mercedes Royuela

royuela@unavarra.es

Specialty section:

This article was submitted to Agroecology and Land Use Systems,

a section of the journal

Frontiers in Plant Science

Received: 04 September 2017 Accepted: 01 November 2017

Published: 16 November 2017

Citation:

Fernández-Escalada $M$, Zulet-González A, Gil-Monreal M, Zabalza A, Ravet K, Gaines T and Royuela M (2017) Effects of EPSPS

Copy Number Variation (CNV) and Glyphosate Application on the Aromatic and Branched Chain Amino Acid Synthesis Pathways in Amaranthus palmeri.

Front. Plant Sci. 8:1970. doi: 10.3389/fpls.2017.01970

\section{Effects of EPSPS Copy Number Variation (CNV) and Glyphosate Application on the Aromatic and Branched Chain Amino Acid Synthesis Pathways in Amaranthus palmeri}

\author{
Manuel Fernández-Escalada ${ }^{1}$, Ainhoa Zulet-González¹, Miriam Gil-Monreal'1, \\ Ana Zabalza', Karl Ravet ${ }^{2}$, Todd Gaines ${ }^{2}$ and Mercedes Royuela ${ }^{1 *}$ \\ 1 Departamento Ciencias del Medio Natural, Universidad Pública de Navarra, Pamplona, Spain, ${ }^{2}$ Department of \\ Bioagricultural Sciences and Pest Management, Colorado State University, Fort Collins, CO, United States
}

A key enzyme of the shikimate pathway, 5-enolpyruvylshikimate-3-phosphate synthase (EPSPS; EC 2.5.1.19), is the known target of the widely used herbicide glyphosate. Glyphosate resistance in Amaranthus palmeri, one of the most troublesome weeds in agriculture, has evolved through increased EPSPS gene copy number. The aim of this work was to study the pleiotropic effects of (i) EPSPS increased transcript abundance due to gene copy number variation (CNV) and of (ii) glyphosate application on the aromatic amino acid (AAA) and branched chain amino acid (BCAA) synthesis pathways. Hydroponically grown glyphosate sensitive (GS) and glyphosate resistant (GR) plants were treated with glyphosate 3 days after treatment. In absence of glyphosate treatment, high EPSPS gene copy number had only a subtle effect on transcriptional regulation of AAA and BCAA pathway genes. In contrast, glyphosate treatment provoked a general accumulation of the transcripts corresponding to genes of the AAA pathway leading to synthesis of chorismate in both GS and GR. After chorismate, anthranilate synthase transcript abundance was higher while chorismate mutase transcription showed a small decrease in GR and remained stable in GS, suggesting a regulatory branch point in the pathway that favors synthesis toward tryptophan over phenylalanine and tyrosine after glyphosate treatment. This was confirmed by studying enzyme activities in vitro and amino acid analysis. Importantly, this upregulation was glyphosate dose dependent and was observed similarly in both GS and GR populations. Glyphosate treatment also had a slight effect on the expression of BCAA genes but no general effect on the pathway could be observed. Taken together, our observations suggest that the high CNV of EPSPS in A. palmeri GR populations has no major pleiotropic effect on the expression of AAA biosynthetic genes, even in response to glyphosate treatment. This finding supports the idea that the fitness cost associated with EPSPS CNV in A. palmeri may be limited.

Keywords: glyphosate, aromatic amino acid pathway, branched chain amino acid pathway, mRNA relative expression, EPSPS, CM, AS, Amaranthus palmeri 


\section{INTRODUCTION}

The shikimate pathway uses carbon from primary metabolism to form chorismate, a precursor of the essential aromatic amino acids (AAAs) phenylalanine (Phe), tyrosine (Tyr), and tryptophan (Trp) (Tzin and Galili, 2010). These AAAs are not only essential components of protein synthesis but also serve as precursors for a wide range of secondary metabolites with multiple biological functions in plants, including plant stress tolerance (Dyer et al., 1989; Keith et al., 1991; Gorlach et al., 1995; Janzik et al., 2005; Maeda and Dudareva, 2012). The AAA synthesis pathway can be subdivided into two steps: (i) the pre-chorismate (shikimate) pathway which provides the precursor chorismate used for synthesis of all AAAs and (ii) the post-chorismate pathway which can lead to either synthesis of Phe and Tyr, or Trp, via different routes (Figure 1) (Maeda and Dudareva, 2012). Synthesis of chorismate is catalyzed by seven enzymes acting sequentially (Figure 1): D-arabino-heptulosonate 7-phosphate synthase (DAHPS), dehydroquinate synthase (DHQS), 3-dehydroquinate dehydratase/shikimate dehydrogenase (DQSD), shikimate kinase (SK), 5-enolypyruvylshikimate 3-phosphate synthase (EPSPS), and chorismate synthase (CS). After formation of chorismate, synthesis of Trp is catalyzed by anthranilate synthase (AS) while synthesis of Phe and Tyr is catalyzed by chorismate mutase (CM) (Tohge et al., 2013).

Due to its importance for plant biology, the synthesis of AAA is a tightly regulated process controlled by many inputs (Bentley and Haslam, 1990; Tzin and Galili, 2010; Tohge et al., 2013; Galili et al., 2016). Four points appear as checkpoints: the entrance of the pathway with the enzyme DAHPS (Sato et al., 2006), an exit of major importance with the phenylalanine ammonia lyase (PAL) (Hahlbrock and Scheel, 1989), the branch point in the post-chorismate pathway (Maeda and Dudareva, 2012) and the enzyme EPSPS. The enzyme EPSPS is the target of the herbicide glyphosate (Steinrücken and Amrhein, 1980) and therefore a key step in the shikimate pathway.

The intensive and continuous use of glyphosate has led to the emergence of glyphosate resistant (GR) weed populations (Powles, 2008). The global issue of herbicide resistance for weed management is a serious challenge for global food security (Délye et al., 2013). One of the most damaging glyphosate-resistant weed species is Amaranthus palmeri S. Wats (Culpepper et al., 2006; Powles and $\mathrm{Yu}, 2010)$. Glyphosate resistance is conferred by gene amplification of EPSPS, which leads to a massive production of the enzyme EPSPS (Gaines et al., 2010). The recommended field dose is not sufficient to inhibit EPSPS activity, and plants survive. Copy number variation (CNV) of EPSPS is now reported to confer glyphosate resistance in several weed species including Lolium multiflorum (Salas et al., 2012) and Kochia scoparia (Wiersma et al., 2015) and particularly in Amaranthus species such as Amaranthus tuberculatus (Lorentz et al., 2014) and Amaranthus spinosus (Nandula et al., 2014).

To date, how the AAA pathway is regulated and how glyphosate may affect this regulation is not clearly understood. In particular, it is unknown whether there are pleiotropic effects associated with EPSPS CNV, particularly at the AAA synthesis pathway. Notably, no fitness cost has been associated with massive increase of EPSPS activity in GR populations (Giacomini et al., 2014; Vila-Aiub et al., 2014). However, the gene amplification resistance mechanism found in A. palmeri offers us the opportunity to study the regulation of the shikimate pathway, the effect of EPSPS overexpression due to extra EPSPS gene copies, and the effect of glyphosate application. In addition to the feedback regulation of AAA biosynthetic pathway, the hypothesis of the existence of cross-regulation of amino acid metabolic pathways at the transcriptional level has been revised (Pratelli and Pilot, 2014). A close correlation between AAA and branched chain amino acids (BCAAs) has been found (Noctor et al., 2002).

In this study, the main objective was to evaluate the impact of EPSPS overexpression by gene amplification and of glyphosate treatment on the regulation of the AAA pathway and free AAA content. To this aim, the response of glyphosate sensitive (GS) and GR populations of $A$. palmeri to glyphosate were evaluated at the molecular and biochemical levels. Additionally, $m$ RNA relative expression of the main enzymes from the BCAA pathway was developed to test whether there is any variation in their levels because of the overexpression of EPSPS or glyphosate treatment.

\section{MATERIALS AND METHODS}

\section{Plant Material and Herbicide Application}

Seeds of A. palmeri GS and GR biotypes were originally collected from North Carolina (United States) (Chandi et al., 2012; Fernández-Escalada et al., 2016). The resistance mechanism of the GR biotype has been described to be EPSPS gene amplification (Chandi et al., 2012), with 47.5 more gene copies in GR than in GS plants (Fernández-Escalada et al., 2016).

Plants were germinated and grown in aerated hydroponic culture under controlled conditions according to procedures described in Fernández-Escalada et al. (2016). Three week-old plants [after reaching the growth stage defined as $\mathrm{BBCH} 14$ (Hess et al., 1997)] were treated with glyphosate (commercial formula, Glyfos, 360 g a.e. $\mathrm{L}^{-1}$, isopropylamine salt, BayerGarden, Valencia, Spain) at both recommended field rate $(1 \times=0.84 \mathrm{~kg}$ $\left.\mathrm{ha}^{-1}\right)$ and three times that rate $\left(3 \times=2.52 \mathrm{~kg} \mathrm{ha}^{-1}\right)$, according to Culpepper et al. (2006). Glyphosate treatment was performed using an aerograph (Junior Start model; Definik; Sagola). Control plants were treated with water. At 3 days after treatment, leaves were collected, frozen, and ground to a fine power as previously described (Fernández-Escalada et al., 2016). The experiment was conducted twice.

\section{Quantitative Reverse Transcription-PCR}

RNA was extracted from leaf tissues using the MacheryNagel NucleoSpin ${ }^{\circledast}$ RNA Plant kit following manufacturer's instructions. Total RNA concentration was measured with Gen 5.1.11 (Biotek Instruments, Inc., United States) and RNA quality was assessed using RNA gel electrophoresis. The gels were visualized using a Gel Doc 2000 system (BIORAD Laboratories, Inc., Hercules, CA, United States). 


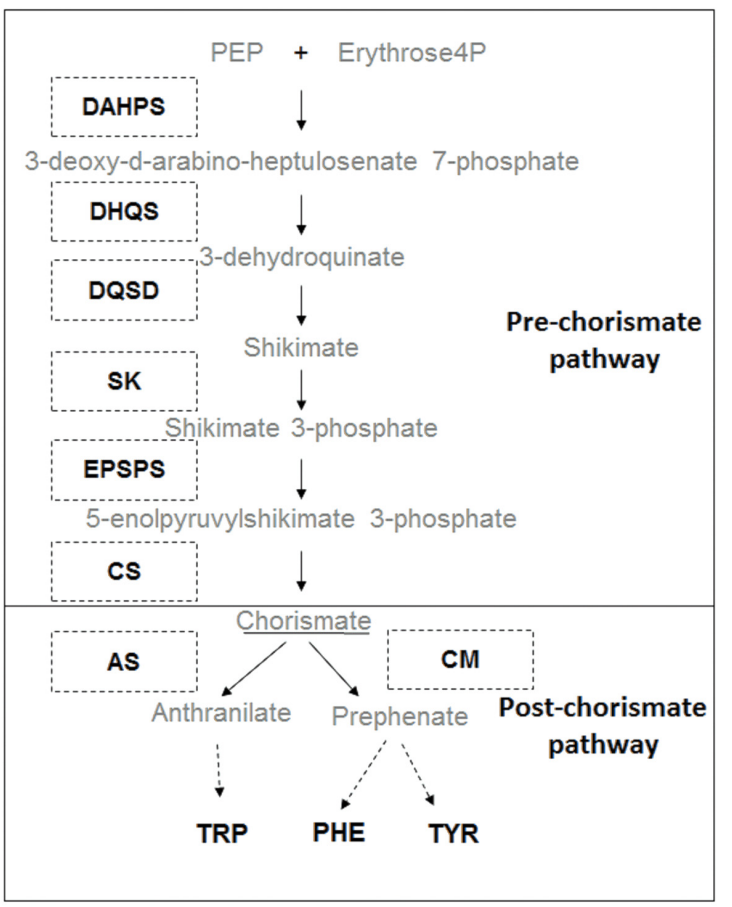

FIGURE 1 | Biosynthetic pathway of aromatic amino acids (AAAs). Consecutive enzymatic steps of pre-chorismate pathway: D-arabino-heptulosonate 7-phosphate synthase (DAHPS), dehydroquinate synthase (DHQS), 3-dehydroquinate dehydratase/shikimate dehydrogenase (DQSD), shikimate kinase (SK), 5-enolypyruvylshikimate 3-phosphate synthase (EPSPS), and chorismate synthase (CS); and post-chorismate pathway: anthranilate synthase (AS) chorismate mutase (CM) leading to the synthesis of tyrosine (TYR), phenylalanine (PHE), and tryptophan (TRP).
cDNA synthesis was performed using BIORAD iScript ${ }^{\mathrm{TM}} \mathrm{cDNA}$ Synthesis Kit with $1 \mu \mathrm{g}$ of total RNA following manufacturer's instructions.

Quantitative RT-PCR (qRT-PCR) was performed using a Thermocycler BIORAD CFX Connect TM Real-Time System. The reaction kit used for qPCR was PerfeCTa SYBR ${ }^{\circledR}$ Green SuperMix (Quantabio, Beverly, MA, United States). Each reaction was performed using $1 \mu \mathrm{L}$ of cDNA template. The following thermal profile was used for all PCRs: denaturation at $95^{\circ} \mathrm{C}$ for $2 \mathrm{~min}, 40$ cycles of $95^{\circ} \mathrm{C}$ for $15 \mathrm{~s}$ and $52-61^{\circ} \mathrm{C}$ for annealing and extension for $20 \mathrm{~s}$. Optimal annealing temperature for each primer was determined using gradient PCR. All primers and annealing temperatures are listed in Supplementary Table 1. EPSPS primer was modified from Gaines et al. (2010). Melting curve analysis was conducted to verify amplification of single PCR products. Gene expression was monitored in five biological replicates. Primer efficiency (E) for each primer is presented in Supplementary Table 1 and was calculated according to $\mathrm{E}=10$ [-1/slope] (Pfaffl, 2001). Relative transcript level was calculated as $\mathrm{E}_{\mathrm{GOI}} \mathrm{CP}_{\mathrm{GOI}}$ control $-\mathrm{CP}_{\mathrm{GOI}}$ treated $/ \mathrm{E}_{\mathrm{REF}} \mathrm{CP}_{\mathrm{REF} \text { control }}-\mathrm{CP}_{\mathrm{REF}}$ treated (Pfaffl, 2001), where GOI = gene of interest, $\mathrm{REF}=$ reference gene (beta tubulin was used as normalization gene), and $\mathrm{CP}=$ crossing point, the cycle at which fluorescence from amplification exceeded the background fluorescence. Relative transcript level was calculated for all genes of the AAA synthesis pathway, corresponding to eight enzymes and four genes of the BCAA synthesis pathway.

\section{EPSPS, DAHPS, and PAL Immunoblotting}

Protein extraction was performed using $0.1 \mathrm{~g}$ of ground leaf tissue in $0.2 \mathrm{~mL}$ of extraction buffer (MOPS $100 \mathrm{mM}$, EDTA 5 mM, Triton-X 100 1\%, glycerin 10\%, KCl $50 \mathrm{mM}$, benzamidine $1 \mathrm{mM}$, iodoacetamide $100 \mu \mathrm{M}$, PVP $5 \%$ and PMSF $1 \mathrm{mM}$ ). Proteins were separated by $12.5 \%$ SDS-PAGE and immunoblots were produced according to standard techniques. The protein amount loaded per well for each antibody used is specified in the figure legends. EPSPS and DAHPS antibody dilutions were 1:2000 (Fernández-Escalada et al., 2016) and 1:1000 (Orcaray et al., 2011), respectively. PAL antibody was produced by a custom peptide facility (Biogenes, Berlin, Germany) using a short, conjugated peptide as an antigen (C-QFAKPR-SDSFEEKN). The antibody was raised in rabbits using standard protocols from the manufacturer, and the primary antibody dilution was 1:500. An anti-rabbit AP conjugated antibody (Sigma Chemical, Co., St. Louis, MO, United States) was used as a secondary antibody at a dilution of 1:20000. Bands were identified using a BCIP/NBT kit which was Amplified alkaline phosphatase immunoblot assay kit (BIORAD 170, BIORAD Laboratories, Inc., Hercules, CA, United States). Immunoblots were scanned using a GS-800 densitometer, and protein bands were quantified using Quantity One software (BIORAD Laboratories, Inc., Hercules, CA, United States). In the case of EPSPS protein, membrane signals were normalized according to total soluble protein loading quantity. In the case of DAHPS and PAL, absolute signals were used.

\section{Enzymatic Activities}

5-Enolpyruvylshikimate-3-phosphate synthase activity was performed using the procedure described in Gaines et al. (2010). PAL activity was carried out according to Orcaray et al. (2011) with the following modifications. Samples were immediately centrifuged after extraction $(12,000 \mathrm{~g}$, $5 \mathrm{~min}$ ) The reaction was started by the addition of $25 \mathrm{mM}$ L-phenylalanine (Maroli et al., 2015). Controls (without L-phenylalanine) were prepared to determine endogenous levels of transcinnamic acid (t-CA). Incubation was performed for $1 \mathrm{~h}$ at $37^{\circ} \mathrm{C}$ (Sarma et al., 1998; Wang et al., 2007).

Protein extraction for CM and AS activity assays was developed as described in Singh and Widholm (1974) with addition of $1 \mathrm{mM}$ PMSF (Goers and Jensen, 1984). Samples were desalted using PD-10 columns (Ishimoto et al., 2010). CM enzymatic activity was measured as described in Goers and Jensen (1984). Control for each sample was carried out using enzymatic extracts previously inactivated with $1 \mathrm{~N} \mathrm{HCl}$. AS activity was quantified as described in Ishimoto et al. (2010). Controls were performed using boiled enzymatic extract (Matsukawa et al., 2002). 


\section{Shikimate Determination}

For shikimate content determination, three leaf disks $(4 \mathrm{~mm}$ diameter) were excised from the youngest leaf of each plant. Leaf disks were placed in a screw-top $2 \mathrm{~mL}$ Eppendorf tube, frozen, and stored at $-80^{\circ} \mathrm{C}$ until analysis. Shikimate was extracted as described in Koger et al. (2005). After addition of $100 \mu \mathrm{L}$ of $0.25 \mathrm{~N} \mathrm{HCl}$ per disk to each vial, samples were incubated at $22^{\circ} \mathrm{C}$ for $1.5 \mathrm{~h}$ and mixed by vortexing. Shikimate content was quantified spectrophotometrically (Cromartie and Polge, 2000).

\section{Aromatic Amino Acid Content Determination}

Ground leaf ( $0.1 \mathrm{~g})$ was homogenized in $1 \mathrm{M} \mathrm{HCl}$ for amino acid extraction. Protein precipitation was performed after incubation on ice and centrifugation (Orcaray et al., 2010). After derivatization with fluorescein isothiocyanate, AAA content was measured by capillary electrophoresis coupled to a laserinduced fluorescence detector, as described in Zulet et al. (2013b). Analyses were performed at $20^{\circ} \mathrm{C}$ and at a voltage of $+30 \mathrm{kV}$. For tryptophan determination, the voltage was reduced to $+20 \mathrm{kV}$ in order to improve separation.

\section{Statistical Analysis}

Transcript level analyses were performed using five biological replicates. For immunoblot, enzyme activity, shikimate and AAA quantification, four biological replicates were used. Oneway ANOVA with a multiple-comparison adjustment for least significant difference (LSD) at $p \leq 0.05$ was used. Statistical analyses were performed using SPSS Statistics 24.0 (IBM, Corp., Armonk, NY, United States).

\section{RESULTS}

The number of EPSPS copies in the studied GR biotype was reported to be 47.5 fold when compared to the corresponding GS biotype (Fernández-Escalada et al., 2016). In the absence of glyphosate, protein level was increased by 25 fold (Figures 2A,B) and EPSPS activity was 26 fold higher (Figure 2C). In response to glyphosate, only a mild increase of the abundance of EPSPS protein was observed in the GR biotype at the highest glyphosate dose (Figures 2A,B). EPSPS activity was not affected by glyphosate in the GR biotype, regardless of the dose, while it was slightly decreased in the GS biotype with the highest dose applied (Figure 2C). While shikimate content was almost negligible in untreated plants of both populations, it accumulated after glyphosate treatment in GS and in GR only at the highest glyphosate dose. Shikimate accumulated significantly more in GS than in GR at each glyphosate dose (Figure 2D), confirming the inhibition of EPSPS by glyphosate observed in GS (Figure 2C).

To study the impact of the high EPSPS copy number on the regulation of the AAA biosynthetic pathway, transcript levels for seven enzymes were analyzed by qRT-PCR. In absence of glyphosate treatment, EPSPS transcript level was increased by 55 fold in GR (Figure 3A), confirming the results of FernándezEscalada et al. (2016). For other enzymes, particularly CS and
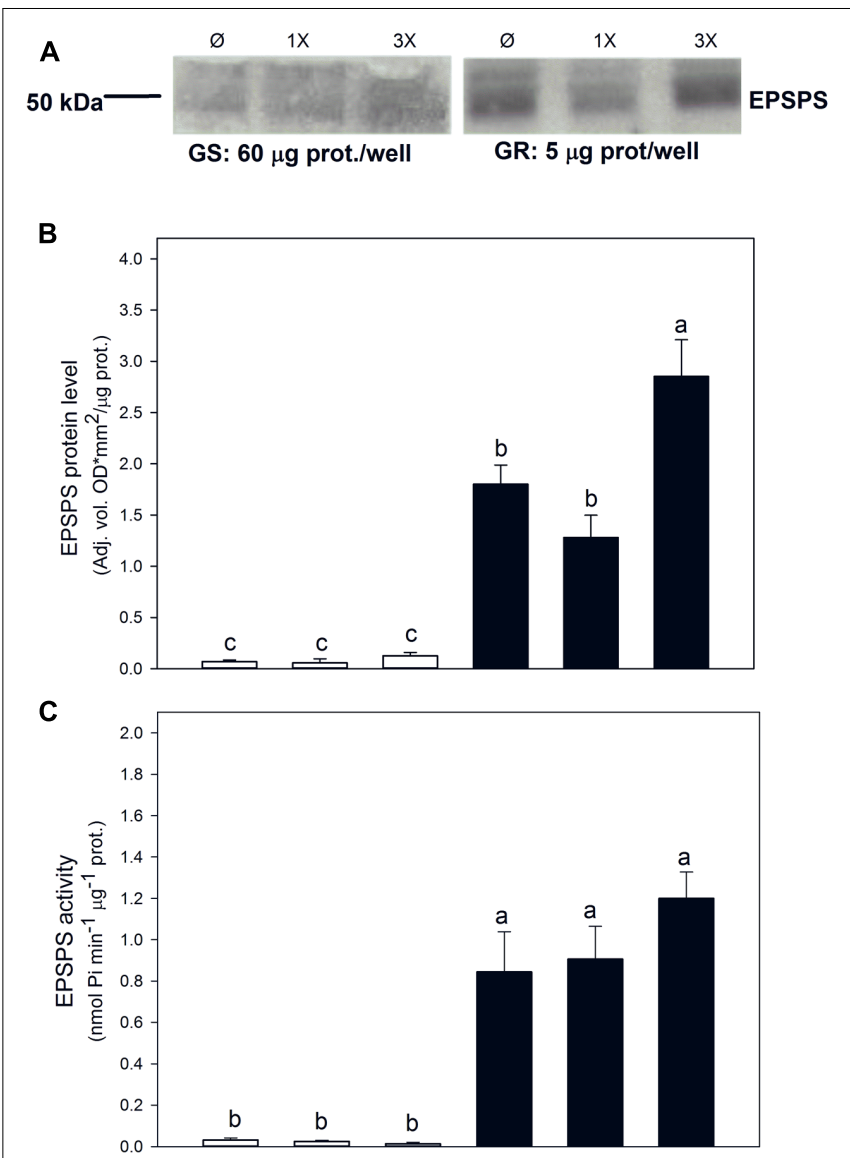

D

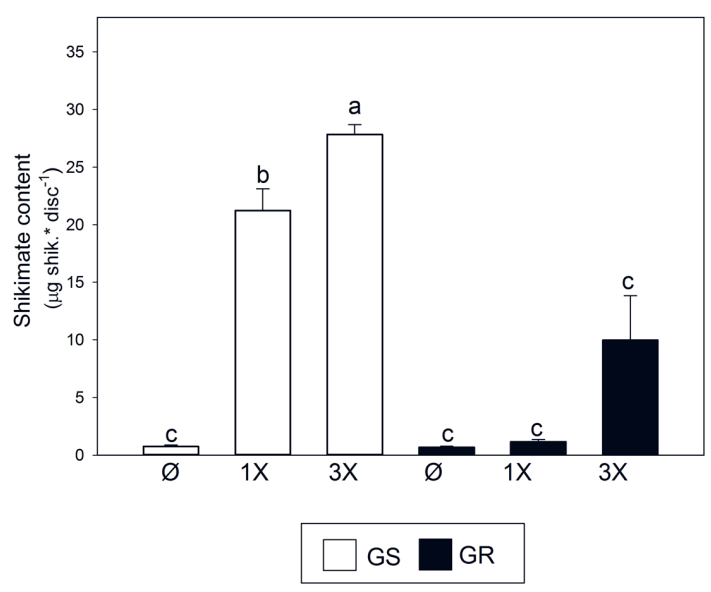

FIGURE 2 | Characterization of resistance in Amaranthus palmeri populations. Glyphosate sensitive (white bars; GS) and glyphosate resistant (black bars; GR) populations were untreated $(\varnothing)$ or treated with glyphosate and measured 3 days after treatment with one (1X) or three times (3X) field dose.

(A) Representative immunoblots for EPSPS. Total soluble proteins (60 $\mu \mathrm{g}$ for GS or $5 \mu \mathrm{g}$ for GR) were fractioned by $12.5 \%$ SDS-PAGE and blotted. (B) Normalization of the intensity of the EPSPS bands expressed as optical density for unit of area per $\mu \mathrm{g}$ of protein (Mean \pm SE; $n=3$ ). (C) EPSPS in vitro enzymatic activity measured spectrophotometrically in semicrude leaf extracts (Mean \pm SE; $n=4$ ). (D) Shikimate content was measured spectrophotometrically after extraction from leaf disks of treated plants (Mean $\pm \mathrm{SE} ; n=4$ ). Different letters indicate significant differences between treatments and/or populations ( $p$-value $\leq 0.05$, LSD test). 
A



B
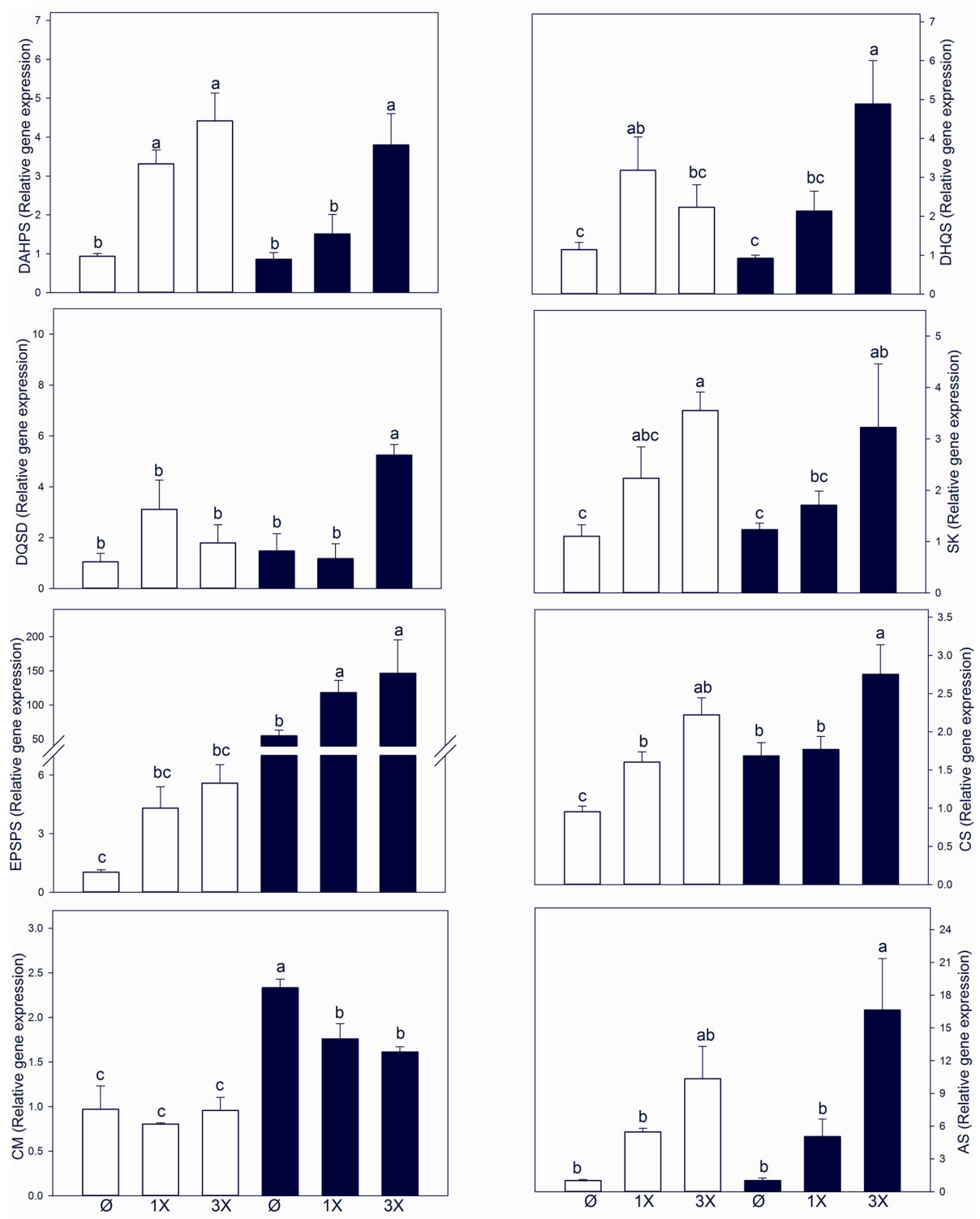

FIGURE 3 | Transcript abundance of genes in the aromatic amino acid (AAA) biosynthetic pathway. Glyphosate sensitive (white bars; GS) and glyphosate resistant (black bars; GR) populations were untreated $(\varnothing)$ or 3 days after treatment with glyphosate at one (1X) or three times (3X) field dose. Enzyme abbreviations as described in Figure 1. (A) Ratio of GR to GS relative transcript abundance measured with qRT-PCR normalized using the normalization gene beta tubulin.

(B) Relative transcript abundance normalized using the normalization gene beta tubulin, relative to GS untreated plants (Mean \pm SE; $n=5$ ). Different letters indicate significant differences between treatments and/or populations ( $p$-value $\leq 0.05$, LSD test).

CM, only marginal changes were observed (1.68 and 2.33 fold, respectively) (Figure 3A).

Glyphosate provoked an induction of the expression of all the genes of the shikimate pathway, with the exception of $C M$ (Figure 3B). The change in gene expression was dose dependent.
The same effect was observed in both GS and GR populations. $C M$ showed the opposite behavior, with no change (GS) or a slight decrease (GR) in CM transcript accumulation after treatment with glyphosate (Figure 3B). The most responsive gene was AS with upregulation over 15 fold in GR with the highest dose 


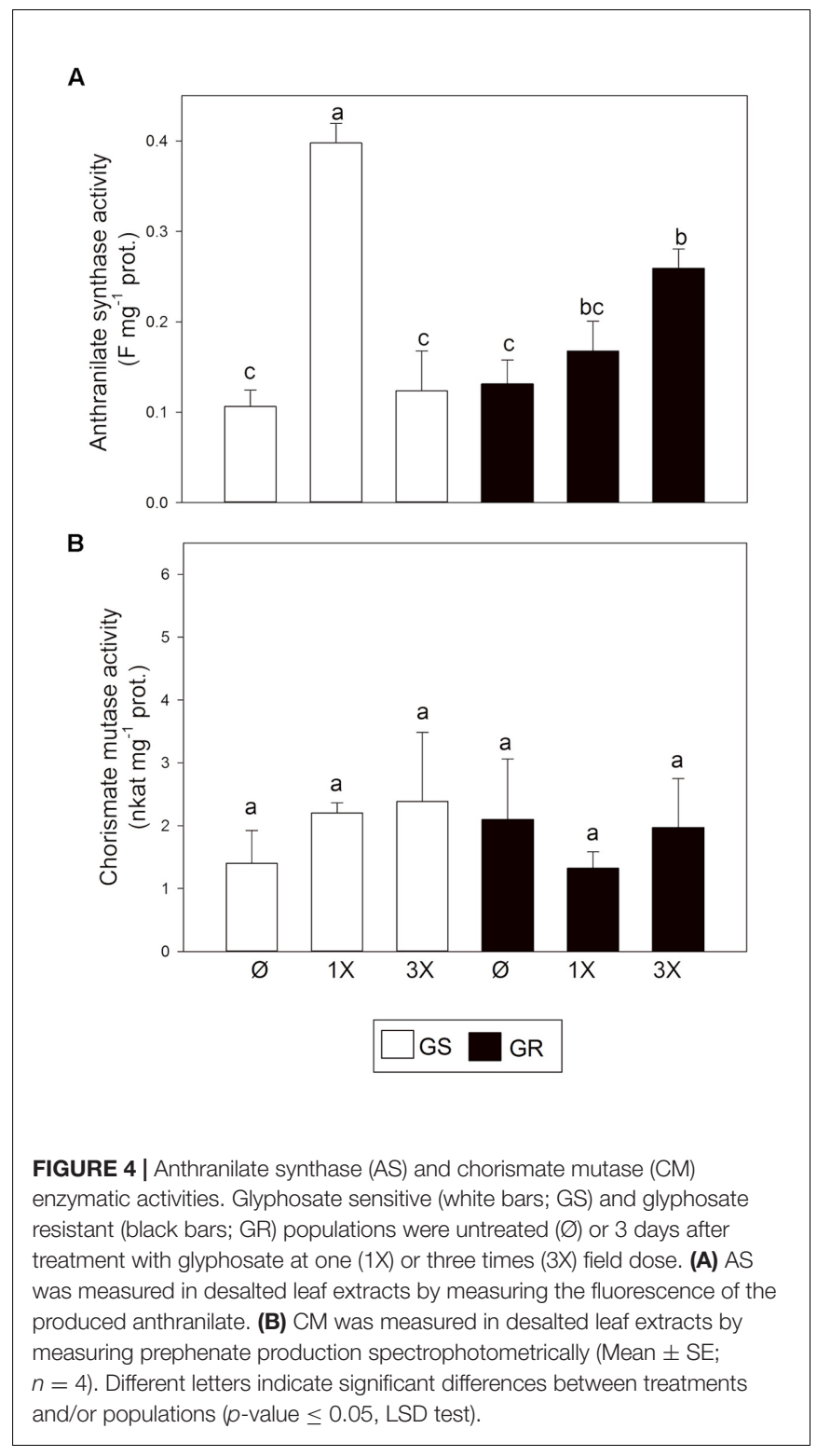

(Figure 3B). This may suggest a preferential flux to the Trp biosynthesis branch rather than to the Phe and Tyr branch in response to glyphosate treatment.

To pursue this hypothesis, the activity of CM and AS enzymes was studied. In the absence of glyphosate, AS (Figure 4A) and $\mathrm{CM}$ (Figure 4B) activities were similar in both biotypes. Changes in the activity of AS and CM confirmed the trend observed at the transcript level, suggesting a preferential synthesis toward Trp after glyphosate treatment. AS expression induction was concomitant with an increase in the enzyme activity while CM activity was unchanged.

Next, AAA levels were measured (Figure 5). Before treatment with glyphosate, levels of Trp (Figure 5A), Tyr (Figure 5B), and Phe (Figure 5C) were similar in both GS and GR biotypes. This result confirms that the striking change in EPSPS expression due to $\mathrm{CNV}$ does not have a major effect on AAA levels.
A

B
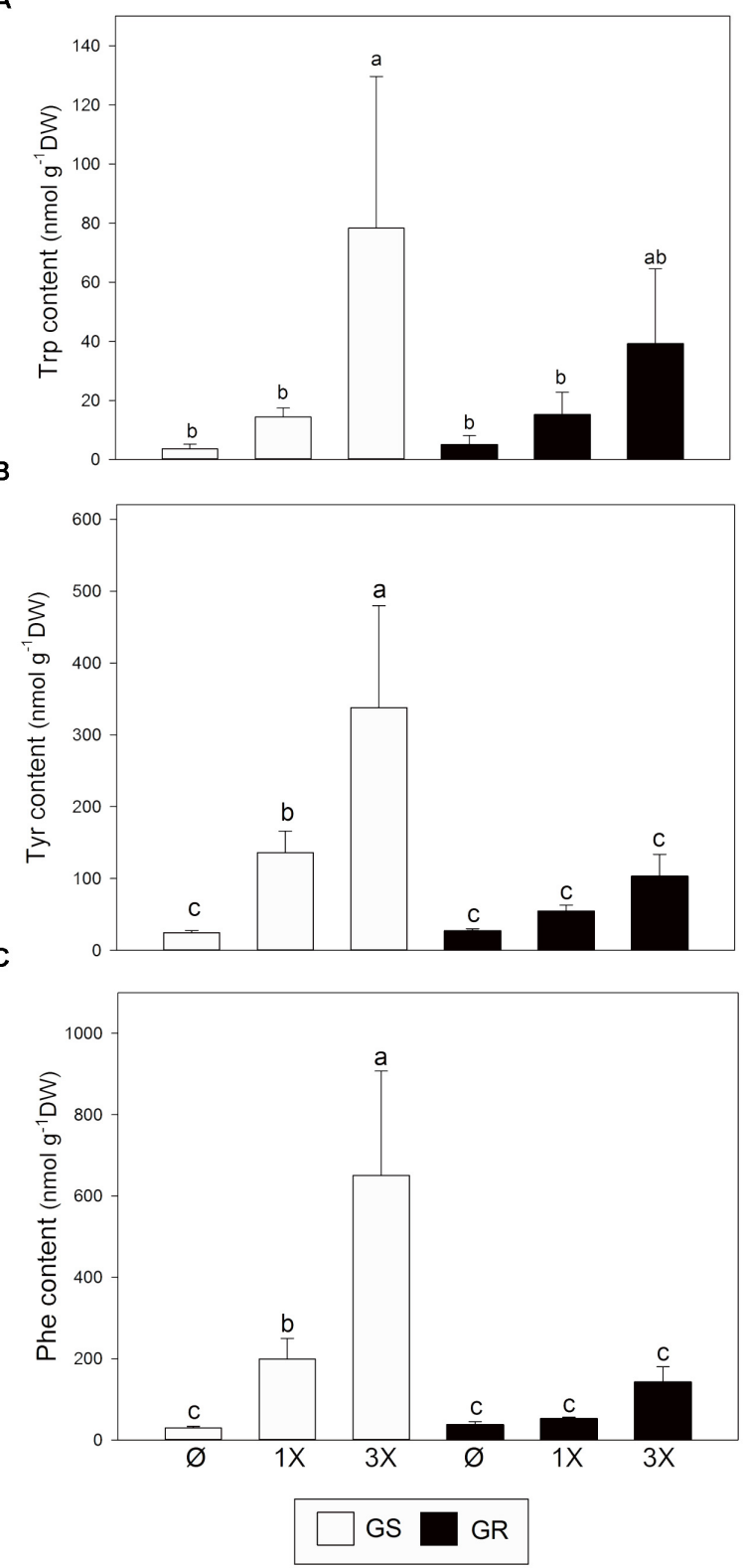

FIGURE 5 | Aromatic amino acid content. Glyphosate sensitive (white bars; GS) and glyphosate resistant (black bars; GR) populations were untreated (Ø) or 3 days after treatment with glyphosate at one (1X) or three times (3X) field dose. Tryptophan (Trp; A), tyrosine (Tyr; B), and phenylalanine (Phe; C) were measured by capillary electrophoresis in leaf acidic extracts (Mean \pm SE; $n=4$ ). Different letters indicate significant differences between treatments and/or populations ( $p$-value $\leq 0.05$, LSD test).

After glyphosate treatment, the level of all AAA increased (Figures 5A-C). However, significant changes were detected only in GS. In GR, the highest increase was detected for Trp.

Previous studies with the same populations and the same time of study and concentration of glyphosate provoked a threefold increase of total free amino acid content and a 12 fold increase of BCAA content (Fernández-Escalada et al., 2016). The higher 
effect of glyphosate on BCAA content than on other amino acid types suggests a possible effect of the herbicide on the BCAA biosynthetic pathway. Based on this, the expression pattern of four enzymes of BCAA biosynthetic pathway was also measured: acetohydroxyacid synthase (AHAS), ketol-acid reductoisomerase (AHAIR), dihydroxyacid dehydratase (DHAD) and branchedchain amino acid transaminase (TA) (Figure 6). Transcript abundance of the BCAA biosynthetic pathway was not different between the untreated plants of both populations, suggesting that EPSPS overexpression does not affect BCAA pathway expression. After glyphosate treatment, AHAS, DHAD, and TA showed no change at either dose in GS or in GR. AHAIR transcript abundance was increased in GS at the highest glyphosate dose, while it did not change in GR after glyphosate treatment.

\section{DISCUSSION}

\section{Characterization of Resistance in \\ A. palmeri Populations}

In the GR population of $A$. palmeri an EPSPS gene amplification (Fernández-Escalada et al., 2016) results in a massive increase of the accumulation of corresponding transcript (Figure 3A) and of the protein level and activity (Figures 2B,C). Our data validate results previously reported in other populations of A. palmeri (Gaines et al., 2010, 2011; Ribeiro et al., 2014), and other weedy plant species such as A. tuberculatus (Lorentz et al., 2014; Chatham et al., 2015), Lolium perenne ssp. multiflorum (Salas et al., 2012), Eleusine indica (Chen et al., 2015), and Kochia scoparia (Wiersma et al., 2015). Additionally our data confirmed the accumulation of shikimate following treatment with glyphosate, mostly in the GS population (Figure 2D). Shikimate is a known stress marker which accumulates following EPSPS inhibition in GS populations (Dyer et al., 1988; Baerson et al., 2002; Zhu et al., 2008; Whitaker et al., 2013; Doğramac1 et al., 2015; Fernández-Escalada et al., 2016; Dillon et al., 2017).

\section{Gene Amplification of EPSPS in A. palmeri GR Populations Has No Major Pleiotropic Effect on the Expression of AAA Biosynthetic Genes}

Despite all these traits that characterize a GR population at molecular and biochemical levels, our work revealed that gene amplification of EPSPS had no major effect on the overall AAA pathway (Figures 2-5). In particular, in untreated plants, the level of free AAA content was similar in GR and GS populations (Figure 5). Similar AAA content in glyphosate resistant/sensitive biotypes has been previously described (Maroli et al., 2015). This is consistent with previous reports suggesting that the overexpression of EPSPS may have no fitness cost in A. palmeri (Giacomini et al., 2014; Vila-Aiub et al., 2014).

The entrance of the primary metabolism to AAA pathway is through DAHPS enzyme (Tohge et al., 2013). Plants control the carbon flux into the pathway by controlling DAHPS transcription and protein abundance (Herrmann and Weaver, 1999). However, it was previously unknown whether GR
A

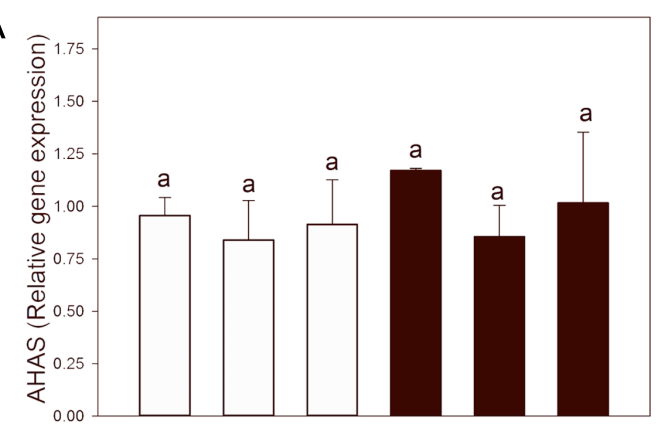

B

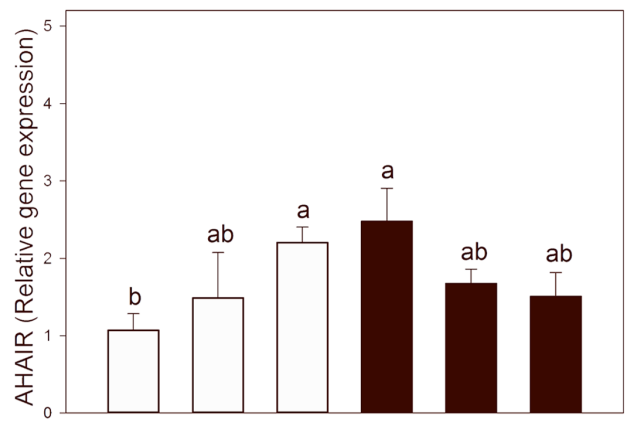

C

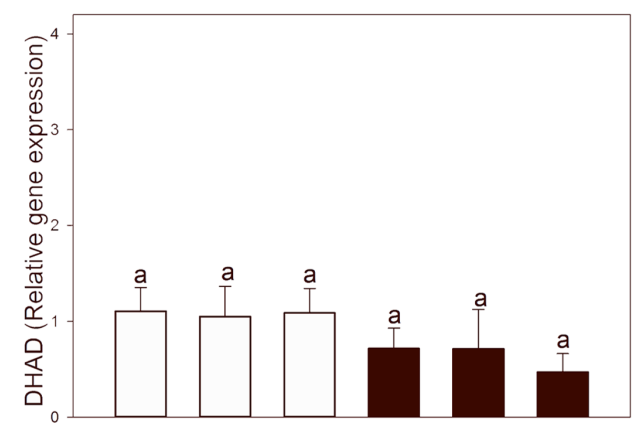

D

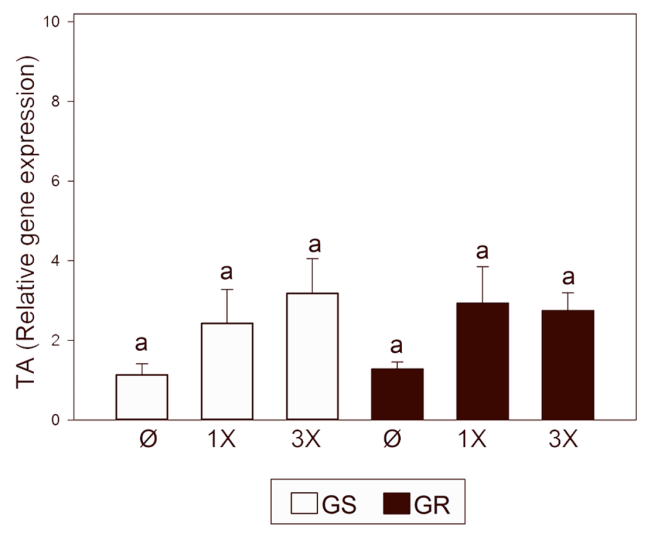

FIGURE 6 | Transcript abundance of genes in the branched chain amino acid (BCAA) biosynthetic pathway. Glyphosate sensitive (white bars; GS) and glyphosate resistant (black bars; GR) populations were untreated $(\varnothing)$ or 3 days after treatment with glyphosate at one $(1 X)$ or three times $(3 X)$ field dose. Relative expression of acetohydroxyacid synthase (AHAS; $\mathbf{A}$ ) ketol-acid reductoisomerase (AHAIR; B), dihydroxyacid dehydratase (DHAD; C) and branched-chain amino acid transaminase (TA; D) normalized with the normalization gene beta tubulin, and relative to untreated GS plants (Mean $\pm \mathrm{SE} ; n=5$ ). Different letters indicate significant differences between treatments and/or populations ( $p$-value $\leq 0.05$, LSD test). 
populations with increased EPSPS expression would have altered DAHPS regulation. Higher levels of DAHPS activity were described in GR populations compared to sensitive populations in Nicotiana tabacum L. (Dyer et al., 1988) and Convolvulus arvensis (Westwood and Weller, 1997). In Lolium rigidum GR populations with higher EPSPS expression, levels of DAHPS transcripts were similar to sensitive population (Baerson et al., 2002). In this study, while DAHPS mRNA relative expression was similar in both populations (Figure 3B), the DAHPS protein level in GR was more than twofold higher than in GS (Supplementary Figures $1 \mathrm{~A}, \mathrm{~B}$ ). It could implicate a translational regulation (or at least post-transcriptional mechanism) that controls DAHPS, and this may be related to EPSPS gene overexpression.

\section{In Sensitive and Resistant Plants Glyphosate Treatment Provokes Increased Transcript Abundance Leading to Synthesis of Chorismate, and after This Regulatory Point, Tryptophan}

Our study shows that glyphosate treatment provoked an accumulation of the transcripts encoding virtually all the enzymes of the shikimate pathway, including EPSPS, in a dose-dependent manner (Figure 3B). This trend seems to be specific for enzymes of the AAA pathway and was not observed for the enzymes of the BCAA pathway (Figure 6). Although increases in some enzymes of the shikimate pathway such as EPSPS (Baerson et al., 2002; Yuan et al., 2002; Chen et al., 2015; Mao et al., 2016) and DAHPS (Baerson et al., 2002) have been previously described, this is the first report suggesting a potential coordinated transcriptional regulation of the shikimate pathway after glyphosate treatment. Because this regulation is observed in both GS and GR populations (Figure 3B), it suggests that this gene upregulation does not occur in response to the level of inhibition of EPSPS activity. Instead, it can be hypothesized that glyphosate itself, or indirectly, may affect plant amino acid metabolism, in addition to its known impact on EPSPS. Future research is needed to determine if glyphosate has unreported effects on plants and what signal causes this general gene induction of the pre-chorismate pathway.

This general upregulation of the expression of genes participating in the pre-chorismate pathway is accompanied with an increase of the accumulation of free AAAs, which is more pronounced in the GS population (Figure 5). Although already reported (Vivancos et al., 2011; Maroli et al., 2015; Fernández-Escalada et al., 2016), this might appear counterintuitive at first glance because glyphosate is inhibiting the entry of carbon in this biosynthetic pathway, and therefore is expected to prevent synthesis of AAA. It is possible that the accumulation of free AAA comes from an increase in protein turnover in the plant following glyphosate treatment (Zabalza et al., 2006; Zulet et al., 2013a; Fernández-Escalada et al., 2016). Isotopic studies in $A$. palmeri revealed that both de novo synthesis of amino acids and protein turnover contribute to AAA accumulation in response to glyphosate (Maroli et al., 2016). While gene expression induction after glyphosate was similar in GR and GS populations (Figure 3B), the accumulation of AAA was mainly observed in GS plants (Figure 5). That observation may suggest that AAA accumulation following glyphosate treatment is rather related to the level of stress experienced by the plant.

After chorismate, $A S$ increase in transcript abundance was higher than any other enzyme in the pathway in response to glyphosate treatment (Figure 3B). AS expression was induced while $C M$ expression was repressed, suggesting a regulatory branch point in the pathway (Figure 1) for a preferential flux of carbon toward Trp biosynthesis over Phe and Tyr biosynthesis. This potential stream toward Trp was confirmed by studying AS and CM enzyme activities in vitro (Figure 4). Data obtained in Arabidopsis thaliana (Sasaki-Sekimoto et al., 2005) and other plant species (Galili et al., 2016) also support this hypothesis. However, measurements of free AAA in treated plants did not reveal any specific accumulation of Trp. Instead all three AAA were accumulated to a similar extent in GS plants (Figure 5). Yet, a slight difference was detected in the GR plants, which may suggest that under "mild" stress ( $3 \mathrm{x}$ dose in GR), synthesis of Trp is prioritized over the synthesis of Phe and Tyr. It is possible that this regulation is related to the inhibition of DAHPS by arogenate (Siehl, 1997), an intermediate product of the CM pathway. DAHPS may be key to the regulation of shikimate synthesis because it represents the entry point in this pathway (Maeda and Dudareva, 2012). Interestingly, DAHPS gene expression was induced by glyphosate in both populations (Figure 3B) while the increase in DAHPS protein was only detected in GS population (Supplementary Figures 1A,B). This might indicate that other layers of regulation (post-transcriptional) might finetune the regulation of this pathway. PAL protein level and enzyme activity have also been studied, because it represents the most important output from the AAA pathway (Hahlbrock and Scheel, 1989). No differences were found between populations for PAL protein abundance or activity level in untreated and treated plants (Supplementary Figures 1C-E). While other studies with other species show important effects of glyphosate on PAL (Hoagland et al., 1979; Zabalza et al., 2017), our results show that PAL abundance and enzyme activity are not affected in A. palmeri.

The results obtained after glyphosate treatment suggest that a stress-induced response to glyphosate increases the enzyme expression in the AAA pathway, which may require a substantial increase in energy consumption (Benevenuto et al., 2017). Trying to increase the carbon flux, which could further increase shikimate accumulation upon glyphosate treatment, could lead to the loss of feedback control in the pathway (Marchiosi et al., 2009). Reduction in AAA levels does not appear to elicit the increased expression of AAA pathway genes, because the AAA concentrations increase with glyphosate dose. Further research is needed to understand the signal(s) that upregulates the AAA pathway following glyphosate treatment.

\section{No Cross Regulation between AAA and BCAA Pathway Was Detected}

In general, the free amino acid pool increases after glyphosate treatment (Orcaray et al., 2010; Vivancos et al., 2011; Zulet et al., 2013a, 2015; Liu et al., 2015) but the higher relative increase 
is in BCAA levels (Orcaray et al., 2010). The higher effect of glyphosate on BCAA than on other amino acid types suggests a possible effect of the herbicide on the BCAA biosynthetic pathway. The expression pattern of the BCAA biosynthetic pathway was measured (Figure 6) and no clear patterns for expression changes of the BCAA enzymes in plants treated with glyphosate were identified (Figure 6), while an induction of expression of AAA enzymes was detected (Figure 3B). Although some authors (Guyer et al., 1995; Noctor et al., 2002; Pratelli and Pilot, 2014) have proposed cross-regulation between the levels of AAA and BCAA, and close correlation was observed between the AAA pathway and the BCAA pathway (Noctor et al., 2002), no cross-regulation at the transcriptional level was found in this study.

\section{CONCLUSION}

No differences were found (other than EPSPS) in transcriptional regulation of the shikimate pathway between $A$. palmeri GR and GS untreated plant, which implies that pleiotropic effects due to shikimate pathway perturbation are not apparent. Transcriptional induction of the AAA pathway was detected following glyphosate treatment in both GR and GS plants, suggesting a potential coordinated transcriptional regulation. AAA content was not the signal causing this response, because AAA accumulation was detected only in GS plants and further research will be needed to determine the signal. Glyphosate treatment resulted in an upregulation of the Trp biosynthesis branch instead of the Phe and Tyr branch, indicating that this branch point may be a regulatory point in the pathway. With respect to cross-regulation between the AAA and BCAA pathways, no differences in BCAA transcriptional regulation were found due to either EPSPS gene amplification or to glyphosate treatment.

\section{REFERENCES}

Baerson, S. R., Rodriguez, D. J., Biest, N. A., Tran, M., You, J., Kreuger, R. W., et al. (2002). Investigating the mechanism of glyphosate resistance in rigid ryegrass (Lolium rigidum). Weed Sci. 50, 721-730. doi: 10.1614/00431745(2002)050[0721:ITMOGR]2.0.CO;2

Benevenuto, R. F., Agapito-Tenfen, S. Z., Vilperte, V., Wikmark, O. G., Van Rensburg, P. J., and Nodari, R. O. (2017). Molecular responses of genetically modified maize to abiotic stresses as determined through proteomic and metabolomic analyses. PLOS ONE 12:e0173069. doi: 10.1371/journal.pone. 0173069

Bentley, R., and Haslam, E. (1990). The shikimate pathway - a metabolic tree with many branches. Crit. Rev. Biochem. Mol. Biol. 25, 307-384. doi: 10.3109/ 10409239009090615

Chandi, A., Milla-Lewis, S. R., Giacomini, D., Westra, P., Preston, C., Jordan, D. L. et al. (2012). Inheritance of evolved glyphosate resistance in a North Carolina Palmer amaranth (Amaranthus palmeri) biotype. Int. J. Agron. 2012:176108. doi: $10.1155 / 2012 / 176108$

Chatham, L. A., Wu, C., Riggins, C. W., Hager, A. G., Young, B. G., Gordon, K., et al. (2015). EPSPS gene amplification is present in the majority of glyphosate-resistant Illinois waterhemp (Amaranthus tuberculatus) populations. Weed Technol. 29, 48-55. doi: 10.1614/WT-D-1400064.1

\section{AUTHOR CONTRIBUTIONS}

$\mathrm{MR}$ and $\mathrm{AZ}$ conceived and designed the experiments, performed by MF-E; KR and MG-M contributed with analysis tools; MF-E, AZ-G, and MG-M prepared figures; MF-E, AZ, $\mathrm{KR}$, TG, and $\mathrm{MR}$ analyzed, discussed results and wrote the manuscript. All authors read and approved the final manuscript.

\section{FUNDING}

MF-E, AZ-G, and MG-M received funding from fellowships trough Universidad Pública de Navarra. This work was financially supported by a grant from the Ministerio Español de Economía y Competitividad (AGL-2016-77531R). This work was also partially financially supported by the USDA National Institute of Food and Agriculture, Hatch project COL00719 to the Colorado State University Agricultural Experiment Station.

\section{ACKNOWLEDGMENTS}

We thank Gustavo Garijo for technical assistance. Any opinions, findings, conclusions, or recommendations expressed in this publication are those of the author(s) and do not necessarily reflect the view of the National Institute of Food and Agriculture (NIFA) or the United States Department of Agriculture (USDA).

\section{SUPPLEMENTARY MATERIAL}

The Supplementary Material for this article can be found online at: https://www.frontiersin.org/articles/10.3389/fpls.2017.01970/ full\#supplementary-material

Chen, J., Huang, H., Zhang, C., Wei, S., Huang, Z., Chen, J., et al. (2015). Mutations and amplification of EPSPS gene confer resistance to glyphosate in goosegrass (Eleusine indica). Planta 242, 859-868. doi: 10.1007/s00425-015-2324-2

Cromartie, T. H., and Polge, N. D. (2000). An improved assay for shikimic acid and its use as monitor for the activity of sulfosate. Proc. Weed Sci. Soc. Am. 4, 121.

Culpepper, A., Grey, T., and Vencill, W. (2006). Glyphosate-resistant Palmer amaranth (Amaranthus palmeri) confirmed in Georgia. Weed Sci. 54, 620-626. doi: $10.1021 /$ jf104719k

Délye, C., Jasieniuk, M., and Le Corre, V. (2013). Deciphering the evolution of herbicide resistance in weeds. Trends Genet. 29, 649-658. doi: 10.1016/j.tig. 2013.06.001

Dillon, A., Varanasi, V. K., Danilova, T. V., Koo, D.-H., Nakka, S., Peterson, D. E., et al. (2017). Physical mapping of amplified copies of the 5-enolpyruvylshikimate-3-phosphate synthase gene in glyphosateresistant Amaranthus tuberculatus. Plant Physiol. 173, 1226-1234. doi: $10.1104 /$ pp.16.01427

Doğramacı, M., Foley, M. E., Horvath, D. P., Hernandez, A. G., Khetani, R. S., Fields, C. J., et al. (2015). Glyphosate's impact on vegetative growth in leafy spurge identifies molecular processes and hormone cross-talk associated with increased branching. BMC Genomics 16:395. doi: 10.1186/s12864-015-1627-9

Dyer, W. E., Henstrad, J. M., Handa, A. K., and Herrmann, K. M. (1989). Wounding induces the first enzyme of the shikimate pathway in Solanaceae. Proc. Natl. Acad. Sci. U.S.A. 86, 7370-7373. doi: 10.1073/pnas.86.19.7370 
Dyer, W. E., Weller, S. C., Bressan, R. A., and Herrmann, K. M. (1988). Glyphosate tolerance in tobacco (Nicotiana tabacum L.). Plant Physiol. 88, 661-666. doi: $10.1104 / p p .88 .3 .661$

Fernández-Escalada, M., Gil-Monreal, M., Zabalza, A., and Royuela, M. (2016). Characterization of the Amaranthus palmeri physiological response to glyphosate in susceptible and resistant populations. J. Agric. Food Chem. 64, 95-106. doi: 10.1021/acs.jafc.5b04916

Gaines, T. A., Shaner, D. L., Ward, S. M., Leach, J. E., Preston, C., and Westra, P. (2011). Mechanism of resistance of evolved glyphosate-resistant Palmer amaranth (Amaranthus palmeri). J. Agric. Food Chem. 59, 5886-5889. doi: $10.1021 /$ jf104719k

Gaines, T. A., Zhang, W., Wang, D., Bukun, B., Chisholm, S. T., Shaner, D. L., et al. (2010). Gene amplification confers glyphosate resistance in Amaranthus palmeri. Proc. Natl. Acad. Sci. U.S.A. 107, 1029-1034. doi: 10.1073/pnas. 0906649107

Galili, G., Amir, R., and Fernie, A. R. (2016). The regulation of essential amino acid synthesis and accumulation in plants. Annu. Rev. Plant Biol. 67, 153-178. doi: 10.1146/annurev-arplant-043015-112213

Giacomini, D., Westra, P., and Ward, S. M. (2014). Impact of genetic background in fitness cost studies: an example from glyphosate-resistant Palmer Amaranth. Weed Sci. 62, 29-37. doi: 10.1614/WS-D-13-00066.1

Goers, S. K., and Jensen, R. A. (1984). The differential allosteric regulation of two chorismate-mutase isoenzymes of Nicotiana sylvestris. Planta 162, 117-124. doi: 10.1007/BF00410207

Gorlach, J., Raesecke, H.-R., Rentsch, D., Regenass, M., Roy, P., Zala, M., et al. (1995). Temporally distinct accumulation of transcripts encoding enzymes of the prechorismate pathway in elicitor-treated, cultured tomato cells. Proc. Natl. Acad. Sci. U.S.A. 92, 3166-3170. doi: 10.1073/pnas.92.8.3166

Guyer, D., Patton, D., and Ward, E. (1995). Evidence for cross-pathway regulation of metabolic gene expression in plants. Proc. Natl. Acad. Sci. U.S.A. 92, 4997-5000. doi: 10.1073/pnas.92.11.4997

Hahlbrock, K., and Scheel, D. (1989). Physiology and molecular biology of phenylpropanoid metabolism. Annu. Rev. Plant Physiol. Plant Mol. Biol. 40, 347-369. doi: 10.1146/annurev.pp.40.060189.002023

Herrmann, K. M., and Weaver, L. M. (1999). The shikimate pathway. Annu. Rev. Plant Physiol. Plant Mol. Biol. 50, 473-503. doi: 10.1146/annurev.arplant.50. 1.473

Hess, M., Barralis, G., and Bleiholder, H. (1997). Use of the extended BBCH scalegeneral for the descriptions of the growth stages of mono- and dicotyledonous weed species. Weed Res. 37, 433-441. doi: 10.1046/j.1365-3180.1997. d01-70.x

Hoagland, R. E., Duke, S. O., and Elmore, C. D. (1979). Effects of glyphosate on metabolism of phenolic-compounds.3. phenylalanine ammonia-lyase activity, free amino-acids, soluble-protein and hydroxyphenolic compounds in axes of dark-grown soybeans. Physiol. Plant. 46, 357-366. doi: 10.1111/j.1399-3054. 1979.tb02633.x

Ishimoto, M., Rahman, S. M., Hanafy, M. S., Khalafalla, M. M., El-Shemy, H. A., Nakamoto, Y., et al. (2010). Evaluation of amino acid content and nutritional quality of transgenic soybean seeds with high-level tryptophan accumulation. Mol. Breed. 25, 313-326. doi: 10.1007/s11032-009-9334-3

Janzik, I., Preiskowski, S., and Kneifel, H. (2005). Ozone has dramatic effects on the regulation of the prechorismate pathway in tobacco (Nicotiana tabacum L. cv. Bel W3). Planta 223, 20-27. doi: 10.1007/s00425-005-0060-8

Keith, B., Dong, X., Ausubel, F. M., and Fink, G. R. (1991). Differential induction of 3-deoxy-D-arabino-heptulosonate 7-phosphate synthase genes in Arabidopsis thaliana by wounding and pathogenic attack. Proc. Natl. Acad. Sci. U.S.A. 88, 8821-8825. doi: 10.1073/pnas.88.19.8821

Koger, C. H., Shaner, D. L., Krutz, L. J., Walker, T. W., Buehring, N., Henry, W. B., et al. (2005). Rice (Oryza sativa) response to drift rates of glyphosate. Pest Manag. Sci. 61, 1161-1167. doi: 10.1002/ps.1113

Liu, Y., Zhang, Y., Liu, Y., Lu, W., and Wang, G. (2015). Metabolic effects of glyphosate on transgenic maize expressing a G2-EPSPS gene from Pseudomonas fluorescens. J. Plant Biochem. Biotechnol. 24, 233-241. doi: 10.1007/s13562-0140263-9

Lorentz, L., Gaines, T. A., Nissen, S. J., Westra, P., Strek, H. J., Dehne, H. W., et al. (2014). Characterization of glyphosate resistance in Amaranthus tuberculatus populations. J. Agric. Food Chem. 62, 8134-8142. doi: 10.1021/jf50 $1040 \mathrm{x}$
Maeda, H., and Dudareva, N. (2012). The shikimate pathway and aromatic amino acid biosynthesis in plants. Annu. Rev. Plant Biol. 63, 73-105. doi: 10.1146/ annurev-arplant-042811-105439

Mao, C., Xie, H., Chen, S., Valverde, B. E., and Qiang, S. (2016). Multiple mechanism confers natural tolerance of three Lily turf species to glyphosate. Planta 243, 321-335. doi: 10.1007/s00425-015-2408-z

Marchiosi, R., Lucio Ferrarese, M. D. L., Bonini, E. A., Fernandes, N. G., Ferro, A. P., and Ferrarese-Filho, O. (2009). Glyphosate-induced metabolic changes in susceptible and glyphosate-resistant soybean (Glycine max L.) roots. Pestic. Biochem. Physiol. 93, 28-33. doi: 10.1016/j.pestbp.2008.09.003

Maroli, A. S., Nandula, V. K., Dayan, F. E., Duke, S. O., Gerard, P., and Tharayil, N. (2015). Metabolic profiling and enzyme analyses indicate a potential role of antioxidant systems in complementing glyphosate resistance in an Amaranthus palmeri biotype. J. Agric. Food Chem. 63, 9199-9209. doi: 10.1021/acs.jafc. 5 b04223

Maroli, A. S., Nandula, V. K., Duke, S. O., and Tharayil, N. (2016). Stable isotope resolved metabolomics reveals the role of anabolic and catabolic processes in glyphosate-induced amino acid accumulation in Amaranthus palmeri biotypes. J. Agric. Food Chem. 64, 7040-7048. doi: 10.1021/acs.jafc.6b02196

Matsukawa, T., Ishihara, A., and Iwamura, H. (2002). Induction of anthranilate synthase activity by elicitors in oats. Z. Naturforsch C 57, 121-128. doi: 10.1515/ znc-2002-1-221

Nandula, V. K., Wright, A. A., Bond, J. A., Ray, J. D., Eubank, T. W., and Molin, W. T. (2014). EPSPS amplification in glyphosate-resistant spiny amaranth (Amaranthus spinosus): a case of gene transfer via interspecific hybridation from glyphosate-resistant Palmer amaranth (Amaranthus palmeri). Pest Manag. Sci. 70, 1902-1909. doi: 10.1002/ps.3754

Noctor, G., Novitskaya, L., Lea, P. J., and Foyer, C. H. (2002). Co-ordination of leaf minor amino acid contents in crop species: significance and interpretation. J. Exp. Bot. 53, 939-945. doi: 10.1093/jexbot/53.370.939

Orcaray, L., Igal, M., Marino, D., Zabalza, A., and Royuela, M. (2010). The possible role of quinate in the mode of action of glyphosate and acetolactate synthase inhibitors. Pest Manag. Sci. 66, 262-269. doi: 10.1002/ps.1868

Orcaray, L., Igal, M., Zabalza, A., and Royuela, M. (2011). Role of exogenously supplied ferulic and p-coumaric acids in mimicking the mode of action of acetolactate synthase inhibiting herbicides. J. Agric. Food Chem. 59, 10162-10168. doi: 10.1021/jf2025538

Pfaffl, M. W. (2001). A new mathematical model for relative quantification in real-time RT-PCR. Nucleic Acids Res. 29, 2002-2007. doi: 10.1093/nar/29.9.e45

Powles, S. B. (2008). Evolved glyphosate-resistant weeds around the world: lessons to be learnt. Pest Manag. Sci. 64, 360-365. doi: 10.1002/ps.1525

Powles, S. B., and Yu, Q. (2010). Evolution in action: plants resistant to herbicides. Annu. Rev. Plant Biol. 61, 317-347. doi: 10.1146/annurev-arplant-042809112119

Pratelli, R., and Pilot, G. (2014). Regulation of amino acid metabolic enzymes and transporters in plants. J. Exp. Bot. 65, 5535-5556. doi: 10.1093/jxb/eru320

Ribeiro, D. N., Pan, Z., Duke, S. O., Nandula, V. K., Baldwin, B. S., Shaw, D. R., et al. (2014). Involvement of facultative apomixis in inheritance of EPSPS gene amplification in glyphosate-resistant Amaranthus palmeri. Planta 239, 199-212. doi: 10.1007/s00425-013-1972-3

Salas, R. A., Dayan, F. E., Pan, Z., Watson, S. B., Dickson, J. W., Scott, R. C., et al. (2012). EPSPS gene amplification in glyphosate-resistant Italian ryegrass (Lolium perenne ssp. multiflorum) from Arkansas. Pest Manag. Sci. 68, 1223-1230. doi: 10.1002/ps.3342

Sarma, A. D., Sreelakshmi, Y., and Sharma, R. (1998). Differential expression and properties of phenylalanine ammonia-lyase isoforms in tomato leaves. Phytochemistry 49, 2233-2243. doi: 10.1016/S0031-9422(98)00336-7

Sasaki-Sekimoto, Y., Taki, N., Obayashi, T., Aono, M., Matsumoto, F., Sakurai, N., et al. (2005). Coordinated activation of metabolic pathways for antioxidants and defence compounds by jasmonates and their roles in stress tolerance in Arabidopsis. Plant J. 44, 653-668. doi: 10.1111/j.1365-313X.2005.02560.x

Sato, K., Mase, K., Nakano, Y., Nishikubo, N., Sugita, R., Tsuboi, Y., et al. (2006). 3-Deoxy-D-arabino-heptulosonate 7-phosphate synthase is regulated for the accumulation of polysaccharide-linked hydroxycinnamoyl esters in rice (Oryza sativa L.) internode cell walls. Plant Cell Rep. 25, 676-688. doi: 10.1007/s00299006-0124-7

Siehl, D. L. (1997). "Inhibitors of EPSP synthase, glutamine synthase and histidine synthesis," in Herbicide Activity: Toxicology, Biochemistry and Molecular 
Biology, eds. R. M. Roe, J. D. Burton, and R. J. Kuhr (Amsterdam: IOS press), 37-67.

Singh, M., and Widholm, J. M. (1974). Measurement of the five enzymes which convert chorismate to tryptophan in wheat plants. Physiol. Plant. 32, 240-246. doi: 10.1111/j.1399-3054.1974.tb03129.x

Steinrücken, H. C., and Amrhein, N. (1980) The herbicide glyphosate is a potent inhibitor of 5-enolpyruvyl-shikimic acid-3-phosphate synthase. Biochem. Biophys. Res. Commun. 94, 1207-1212. doi: 10.1016/0006-291X(80)90547-1

Tohge, T., Watanabe, M., Hoefgen, R., and Fernie, A. R. (2013). Shikimate and phenylalanine biosynthesis in the green lineage. Front. Plant Sci. 4:62. doi: $10.3389 /$ fpls.2013.00062

Tzin, V., and Galili, G. (2010). New insights into the shikimate and aromatic amino acids biosynthesis pathways in plants. Mol. Plant 3, 956-972. doi: 10.1093/mp/ ssq048

Vila-Aiub, M. M., Goh, S. S., Gaines, T. A., Han, H., Busi, R., Yu, Q., et al. (2014). No fitness cost of glyphosate resistance endowed by massive EPSPS gene amplification in Amaranthus palmeri. Planta 239, 793-801. doi: 10.1007/ s00425-013-2022-x

Vivancos, P. D., Driscoll, S. P., Bulman, C. A., Ying, L., Emami, K., Treumann, A., et al. (2011). Perturbations of amino acid metabolism associated with glyphosate-dependent inhibition of shikimic acid metabolism affect cellular redox homeostasis and alter the abundance of proteins involved in photosynthesis and photorespiration. Plant Physiol. 157, 256-268. doi: 10.1104/ pp.111.181024

Wang, Y., Chen, J.-Y., Jiang, Y.-M., and Lu, W.-J. (2007). Cloning and expression analysis of phenylalanine ammonia-lyase in relation to chilling tolerance in harvested banana fruit. Postharvest Biol. Technol. 44, 34-41. doi: 10.1016/j. postharvbio.2006.11.003

Westwood, J. H., and Weller, S. C. (1997). Cellular mechanisms influence differential glyphosate sensitivity in field bindweed (Convolvulus arvensis) biotypes. Weed Sci. 45, 2-11.

Whitaker, J. R., Burton, J. D., York, A. C., Jordan, D. L., and Chandi, A. (2013). Physiology of glyphosate-resistant and glyphosate-susceptible Palmer amaranth (Amaranthus palmeri) biotypes collected from North Carolina. Int. J. Agron. 2013:429294. doi: 10.1155/2013/429294

Wiersma, A. T., Gaines, T. A., Preston, C., Hamilton, J. P., Giacomini, D., Robin Buell, C., et al. (2015). Gene amplification of 5-enol-pyruvylshikimate3-phosphate synthase in glyphosate-resistant Kochia scoparia. Planta 241, 463-474. doi: 10.1007/s00425-014-2197-9
Yuan, C. I., Chaing, M. Y., and Chen, Y. M. (2002). Triple mechanisms of glyphosate-resistance in a naturally occurring glyphosate-resistant plant Dicliptera chinensis. Plant Sci. 163, 543-554. doi: 10.1016/S0168-9452(02) 00147-4

Zabalza, A., Gaston, S., Ribas-Carboì, M., Orcaray, L., Igal, M., Royuela, M., et al. (2006). Nitrogen assimilation studies using $15 \mathrm{~N}$ in soybean plants treated with imazethapyr, an inhibitor of branched-chain amino acid biosynthesis. J. Agric. Food Chem. 54, 8818-8823. doi: 10.1021/jf061 8224

Zabalza, A., Orcaray, L., Fernández-escalada, M., Zulet-gonzález, A., and Royuela, M. (2017). The pattern of shikimate pathway and phenylpropanoids after inhibition by glyphosate or quinate feeding in pea roots. Pestic. Biochem. Physiol. 141, 96-102. doi: 10.1016/j.pestbp.2016.12.005

Zhu, J., Patzoldt, W., and Shealy, R. (2008). Transcriptome response to glyphosate in sensitive and resistant soybean. J. Agric. Food Chem. 56, 6355-6363. doi: 10.1021/jf801254e

Zulet, A., Gil-Monreal, M., Villamor, J. G., Zabalza, A., van der Hoorn, R. A. L., and Royuela, M. (2013a). Proteolytic pathways induced by herbicides that inhibit amino acid biosynthesis. PLOS ONE 8:e73847. doi: 10.1371/journal. pone. 0073847

Zulet, A., Gil-Monreal, M., Zabalza, A., van Dongen, J. T., and Royuela, M. (2015). Fermentation and alternative oxidase contribute to the action of amino acid biosynthesis-inhibiting herbicides. J. Plant Physiol. 175, 102-112. doi: 10.1016/ j.jplph.2014.12.004

Zulet, A., Zabalza, A., and Royuela, M. (2013b). Phytotoxic and metabolic effects of exogenous quinate on Pisum sativum L. J. Plant Growth Regul. 32, 779-788. doi: $10.1007 / \mathrm{s} 00344-013-9345-5$

Conflict of Interest Statement: The authors declare that the research was conducted in the absence of any commercial or financial relationships that could be construed as a potential conflict of interest.

Copyright (c) 2017 Fernández-Escalada, Zulet-González, Gil-Monreal, Zabalza, Ravet, Gaines and Royuela. This is an open-access article distributed under the terms of the Creative Commons Attribution License (CC BY). The use, distribution or reproduction in other forums is permitted, provided the original author(s) or licensor are credited and that the original publication in this journal is cited, in accordance with accepted academic practice. No use, distribution or reproduction is permitted which does not comply with these terms. 